\title{
Study of neonatal morbidity and mortality patterns in a referral tertiary care neonatal unit of a teaching hospital
}

\author{
Khuram $\mathrm{SA}^{1}$, Prasad $\mathrm{CN}^{2}$ \\ ${ }^{1}$ Dr. Mir Sumsam Ali Khurram, Associate Professor, Department of Pediatrics, Deccan College of Medical Sciences, \\ Hyderabad, Telangana State, India, ${ }^{2}$ Dr. C.N. Prasad, Professor \& Head of the Department of Pediatric Surgery, Deccan \\ College of Medical Sciences, Hyderabad, Telangana State, India.
}

Address for Correspondence: Mir Sumsam Ali Khurram, Department of Pediatrics, Deccan College of Medical Sciences, P.O Box Kanchan Bagh, DMRL'X' Road, Santosh Nagar, Hyderabad, Telangana State, India. E-mail :dr.sakhurram@gmail.com

\begin{abstract}
Objective: To find out the incidences and causes of neonatal morbidity and mortality. The present study is carried out with the objective of finding out the causes of neonatal morbidity and mortality at the referral neonatal unit of teaching hospital which caters to the neonatal deliveries in the hospital and the cases brought directly or referred from the smaller hospitals in the region. Methods: A hospital based descriptive study was conducted from January 2014 to December 2014. Each case was studied with reference to maternal / neonatal factors affecting the neonatal mortality and morbidity. The maternal factors studied were maternal age, parity, antenatal care, presentation, mode of delivery, medical illness and obstetric complications. Neonatal factors include sex of the babies, birth weight and gestational age. Result: Total admissions of neonates during the study period were 1169, out of which 385 were intramural (inborn) \& 784 extra mural (out born). Approximately, 37.90\% children were of low birth weight and $25.40 \%$ were preterm babies. HMD, birth asphyxia, septicemia, prematurity, hyperbilirubinemia, congenital malformations were the chief morbidities. The main causes of neonatal mortality were perinatal hypoxia (40.09\%), infection $(27.64 \%)$, Hyaline membrane disease $(12.09 \%)$ and immaturity $(8.29 \%)$. Conclusion: Neonatal mortality rate was $12.94 \%$ which was influenced by sex of the baby. Birth asphyxia tops the list followed by infection as the cause of neonatal morbidity and mortality.
\end{abstract}

Keywords: Mortality, Neonates, Premature babies.

\section{Introduction}

Since the incidences of neonatal problems vary in different countries, It is essential to have data on the local patient population for planning a neonatal service of a maternity unit [1]. These data can help in choosing the priorities in babies weight $(<2500 \mathrm{~g})$ accounting for a high rate of morbidity and mortality [2]. Every year, 8 million low birth weight are born in India [3]. It is projected that 1 million babies suffer from birth asphyxia [4], respiratory distress syndrome and hyperbilirubinemia, while 0.5 million babies, showed evidence of neonatal morbidity and mortality.

Manuscript received: $25^{\text {th }}$ March 2016

Reviewed: $7^{\text {th }}$ April 2016

Author Corrected; $20^{\text {th }}$ April 2016

Accepted for Publication: $1^{\text {st }}$ May 2016
Data on causes of neonatal morbidity and mortality in a referral neonatal unit receiving home delivered neonates from the community as well as from the first referred unit in the government and private sector was lacking.

The high incidences of low birth weight babies in India were due to neglect of nutrition, health and education of women in society. Early teenage marriage (60\%) frequent pregnancies, maternal malnutrition, anemia and infection were important contributory causes [5].

Neonatal mortality rate as reported from various parts of India ranges from 17.9-31.0/1000 live birth. The causes of perinatal and neonatal deaths were often 
presented as maternal, obstetric and fetal [6]. In order to evolve effective strategies to improve perinatal survival, it was obligatory to identify leading causes of perinatal deaths. Neonatal morbidity of a significant nature follows the same pattern as mortality [7].

The lowest illness rate was $4 \%$. The present study was carried out with the objective of finding out the causes of neonatal morbidity and mortality at the neonatal unit of the hospital catering to neonates and the cases brought directly or referred from smaller hospitals in the region. [8].

\section{Materials and Methods}

This hospital-based descriptive study was conducted over a period of 12 months from January 2014 to December 2014 in the neonatal unit of Owaisi Teaching Hospital attached to Deccan College of Medical Sciences, Hyderabad, Telangana State, India. This hospital caters to booked, un booked and high risk pregnant mothers referred from neighboring hospital.

Inclusive Criteria: All new born babies brought alive admitted in neonatal unit.

Exclusive Criteria: All the new bornbabies brought dead to the neonatal unit were excluded from the study.
The hospital have 16 radiant warmers with ventilator support, phototherapy units, and facilities for exchange transfusion. Each case was studied with reference to the maternal / neonatal factors affecting the neonatal morbidity and mortality.

All the babies whose mothers were uncertain about the data of last menstrual period were assessed by modified scoring system of gestation.

Data was collected in a predesigned Proforma showing about the place of birth, Sexes, weight, age at admission, gestational age and mode of delivery. On admission in NICU, the new born were examined by doctors and then by neonatologist. Sample collection involved the selection of elements based on birth weight and gestational age. Diagnosis was mainly clinical and based on WHO definition for prematurity, low birth weight and very low birth weight.

Low birth weight was defined as weight $<2500 \mathrm{gm}$. Statistical analysis was performed using Graph pad prism 5 (Graph pad software Inc. U.S.A). Data is presented as mean +S.D. Variable difference with $\mathrm{P}<0.05$ is considered statistically significant. The sample size was determined by using the Open EPI Statistics and $95 \%$ of confidence was used to detect the results with $90 \%$ of sample power.

\section{Results}

Present study comprised 1676 live births in Owaisi Teaching Hospital, Hyderabad Telangana State India from January 2014 to December 2014. In the present study, the total cases admitted in NICU were 1169. Out of these, 385 were intramural (inborn) and 784 were extramural (out born). Mortality in inborn \& out born deliveries were 61 \& 156 respectively. The present study shows that birth asphyxia developed in 286 neonates an incidence of $17.00 \%$ in which 167 babies I,e $9.96 \%$ were suffering from stage- II and 119 babies i.e. $7.10 \%$ were suffering fromstage- 111 . Thusbirth asphyxia is the leading cause of morbidity and mortality.

Table-1: Neonatal morbidity and mortality.

\begin{tabular}{|c|c|c|}
\hline & No. of Cases & Percentage \\
\hline Live birth & 1676 & \\
\hline Morbidity & 952 & 56.80 \\
\hline Over 2000 gm & 455 & 27.11 \\
\hline Below 2000 gm & 181 & 10.79 \\
\hline Mortality & 217 & 12.94 \\
\hline Over 2000 gm & 160 & 9.54 \\
\hline Below 2000 gm & 57 & 3.40 \\
\hline
\end{tabular}


Table 1 shows morbidity and mortality in babies weighing less than 2000 gm which was much higher as compared with those babies weighing more than 2000 gm.

The mortality was $12.94 \%$ and morbidity was $56.80 \%$ when weight was $1000 \mathrm{gm}$ to $1500 \mathrm{gm}$.

Table-2: Causes of Respiratory distress syndrome.

\begin{tabular}{|c|c|c|}
\hline Causes & No. of Cases & Percentage \\
\hline HMD & 65 & 27.89 \\
\hline Transient tachypnea of new born & 16 & 6.86 \\
\hline Meconium aspiration syndrome & 125 & 53.64 \\
\hline Congenital heart disease & 12 & 5.15 \\
\hline Congenital malformation & 15 & 6.43 \\
\hline Total & $\mathbf{2 3 3}$ & \\
\hline
\end{tabular}

Table 2 shows that causes of respiratory distress in which HMD was found in 65 neonates i.e. 27.89\%, transient tachypnea of a new born was 16 i.e $6.86 \%$ meconium aspiration syndrome was present in 125 (53\%) neonates. Congenital heart diseases were found in 12 babies and congenital malformation was detected in 15 babies.

Table-3: Causes of neonatal hyperbilirubinemia.

\begin{tabular}{|c|c|c|}
\hline Neonatal hyperbilirubinemia & No. of Cases & Percentage \\
\hline Prematurity & 110 & 76.38 \\
\hline ABO incompatibility & 18 & 12.50 \\
\hline Rh incompatibility & 4 & 2.77 \\
\hline Sepsis & 4 & 2.77 \\
\hline Total & 144 & \\
\hline
\end{tabular}

Table 3 shows the causes of neonatal hyperbilirubineamia In our study prematurity was the highest i.e $76.38 \%$. ABO incompatibility was the second leading cause.

Table- 4: Neonatal morbidity systemic sepsis.

\begin{tabular}{|c|c|c|}
\hline Diagnosis & No. of Cases & Percentage \\
\hline Septicemia & 276 & 61.60 \\
\hline Culture positive & 131 & 29.24 \\
\hline Pneumonia & 28 & 6.25 \\
\hline Other & 13 & 2.90 \\
\hline Total & $\mathbf{4 4 8}$ & \\
\hline
\end{tabular}

Table 4 Shows that out of 1676 cases 276 cases were of septicemia (61.60\%) and 131 cases were of positive culture (29.24\%) which constituted a major cause of morbidity. There were 28 cases of pneumonia $(6.25 \%)$.

Table- 5: Maternal age and mortality.

\begin{tabular}{|c|c|}
\hline Age group & Live Birth \\
\hline$<18$ & 70 \\
\hline $19-35$ & 1544 \\
\hline$>35$ & 62 \\
\hline Total & 1676 \\
\hline
\end{tabular}


Table 5 shows maternal age and mortality. Majority of the mothers were in the age group 19-35 years and others were in the age group $<18$ years and above 35 years. The neonatal mortality was the highest in mothers $<18$ years of age.

Table- 6: Presentation and Neonatal mortality.

\begin{tabular}{|l|c|c|c|c|}
\hline \multirow{2}{*}{ Presentation } & \multicolumn{2}{|c|}{ Live Birth } & \multicolumn{2}{c|}{ Neonatal deaths } \\
\cline { 2 - 5 } & No & Percent & 175 & 80.64 \\
\hline Vertex & 1604 & 95.70 & 32 & 14.75 \\
\hline Breech & 51 & 3.04 & 8 & 3.68 \\
\hline Transverse lie & 15 & 0.89 & 2 & 0.94 \\
\hline Cord Prolapse & 6 & 0.35 & $\mathbf{2 1 7}$ & \\
\hline Total & $\mathbf{1 6 7 6}$ & & & Percent \\
\hline
\end{tabular}

Table 6 Shows that the mode of presentation in majority of births were vertex. Breech, transverse lie and prolapse constituted $4.29 \%$.

\section{Discussion}

The main source of information regarding neonatal mortality and morbidity was obtained from hospital where only referred patients with high risk and consistent antenatal records are admitted. The spectrum takes into account severity of neonatal morbidity, details of the newborn and obstetric back ground of the mother [9].

The present study shows that the morbidity in babies weighing less than $2000 \mathrm{gm}$ at birth was higher as compared with those babies weighing more than $2000 \mathrm{gm}$. In the study, neonatal morbidity in babies <2000 gm was $10.76 \%$ while that in Gupta et al reported $24.7 \%$ which is higher than the above observation [10].

The present study shows that the leading causes of neonatal morbidity are birth asphyxia which account for $17.06 \%$, respiratory distress syndrome $6.14 \%$, neonatal hyperbilirubinemia $8.59 \%$, septicemia $16.46 \%$ and congenital malformation 2.08 .

Further analysis of our study shows that $7.06 \%$ of morbidity studied by other workers reported birth asphyxia as a major cause of morbidity [11].

Birth asphyxia was as the main cause for morbidity reported by Singh et.al. and Gupta et.al. in 5.9\% and $2.5 \%$ cases respectively [12]. Bhakoo et.al. [13] and Kapoor et. al [14] however reported these figures as 5.9\% and 5.7\% in their study. Our study shows a higher rate of morbidity in comparison with other studies [15].

Data in our hospital was collected from both booked and un-booked cases and revealed that $6.14 \%$ of the cases were affected with respiratory distress syndrome for morbidity. This figure is higher than what was obtained by Bhargava et.al $2.3 \%$. [16]. This difference in morbidity is significant [16].

The incidences septicemia were the lowest in our study recorded at $16.46 \%$ when compared with the figures quoted from earlier studies [17].

Our study shows that Staphy lococcus aureus is the predominant organism isolated in blood culture followed by Cons, Ecoli. Abhay T Bang et.al [18] reported Ecoli in 37.5\%, Staphylococcus aureus in 29.5\%, Kleisbiella in $20.80 \%$ and Pseudomonas in $12.5 \%$ of samples. 
The present study accounts for $2.44 \%$ of morbidity. The study conducted by Singh et.al reports congenital malformation at $5 \%$. Our study shows the lowest rate of mortality at $12.94 \%$ in comparison with figures collected from different parts of the country.

Besides, our study also shows low neonatal mortality at 12.9 per 1000 live births in comparison with other studies [19] which reported these figures in the range of 18 to 31.

Table- 7: Neonatal mortality Rate A Comparison.

\begin{tabular}{|l|c|c|}
\hline Authors & Year & Neonatal Mortality Rate \\
\hline Babu Usman Ahmedu et al [1] & 2013 & $27.7 \%$ \\
\hline Kaushik et al [2] & $1994-95$ & $31.02 \%$ \\
\hline Hoang t teram et al[7] & 2012 & $19.2 \%$ \\
\hline Clacifweiric et al[8] & 2005 & $18.6 \%$ \\
\hline C. Klingenberg et al [11] & 2003 & $19 \%$ \\
\hline Tallat-seyal et al [19 & 2011 & $30.9 \%$ \\
\hline Gupta Pk et al [20] & $1978-82$ & $28.40 \%$ \\
\hline Kameswaram et al[24] & 1990 & $22.70 \%$ \\
\hline Pradeep et al [25] & $1992-93$ & $18.30 \%$ \\
\hline Present study & $2014-2015$ & $12.94 \%$ \\
\hline
\end{tabular}

In our study on neonatal mortality in mothers in the age group of less than 18 years was $14.28 \%$ whereas in the age group 19-35 years and more showed $12.89 \%$. There was a significant difference in neonatal mortality between maternal age less than 18 years and more than 18 years. In our study, the mortality was high compared with the figures given by Gupta et al [20]. It was also noted that mother below 20 years and more than 35 years, were at risk for higher incidences of neonatal mortality [21]. The present study shows that the mortality was less in comparison with Choudhary et al [22].

The present study shows that neonatal mortality rates in relation to parity were $19.3 \%, 13.80 \%$ and $6.52 \%$ in Primigravida, gravida 2-4 and gravida 5 and above respectively. Similar observation was made by Mavelankar et al [23] that parity was affected on neonatal mortality rate. However, Choudhry et al observed high neonatal mortality of $6 \%$ in primi mothers.

In our case, statistical differences in mortality rates in un booked cases were high when compared with booked cases. In booked cases the mortality rate was $9.51 \%$ and in un booked cases it was $17.30 \%$ [14]. The present study shows that more than half of the neonatal deaths are associated with obstetric complications, PIH being the major complication resulting in $28.27 \%$ of deaths of which $4.76 \%$ were due to eclampsia. It was implied from this observation that PIH increases the risk of neonatal mortality by three folds. Since in our study more than half of neonatal deaths were associated with obstetric complications, it is easy to establish the need for regular antenatal checkup for early diagnosis of these complications and their management.

The present study also shows that neonatal mortality was high in breech and cord prolapsed. The statistical analysis shows a significant difference in mortality rate between vertex presentation and presentation other than the vertex. Neonatal mortality in our study was similar for both male and female babies. Our observation was in agreement with that of kameswaram et al [24].

The neonatal mortality in the present study in birth weight of babies $<1250$ gm was $72.22 \%$ and for those with birth weight $1251-1750 \mathrm{gm}$ was $37.50 \%$. This shows that there are statistical differences in mortality based on the birth weight of the babies as well. Kamehswaram et al reported mortality of 76.9\% [24]. Neonatal mortality in our study for the babies with birth weight between 1501-2000gm was $9.17 \%$ which is comparable to $8.5 \%$ mortality reported by Pradeep et al [25]. 
However, Chavan et al [26] reported high mortality rate i.e. $34.6 \%$ for the same group. The neonatal mortality in babies of more than 2000 gm weight was $2.39 \%$ while Pradeep et at[25] record it at $0.96 \%$ and Chavan et al [26] estimate $4.82 \%$ which is higher than both the observations cited above.

Therefore we may conclude that to reduce neonatal mortality further, those babies whose birth weight is $<1500 \mathrm{gm}$ need better perinatal care. It is observed that in our study there was a decrease in the gestational age i.e $<28$ week from $90.32 \%$ to $64.28 \%$ [27]. The difference in mortality rate of these two groups was statistically significant. Similarly, neonatal mortality for $31-32$ weeks of gestation age was $41.30 \%$ and $33-34$ weeks gestation age was $37.68 \%$ which was also statistically significance.

The present study shows that mortality in babies with more than 32 weeks of gestation period was 10 times less than that reported by Pradeep et al [25]. Overall neonatal mortality in preterm babies of <32 weeks of gestation was less. Our study shows $46.59 \%$ which is higher than $34.7 \%$ and $29.7 \%$ as reported by Chavanet al [26].

In our study, the leading cause of neonatal death is perinatal hypoxia (including birth trauma) accounting for $40.09 \%$ followed by infection $27.67 \%$, HMD is $12.95 \%$, immaturity is $8.29 \%$ miscellaneous was $6.45 \%$ and unknown was $4.60 \%$.

The study by other workers reported birth asphyxia as the leading cause of neonatal mortality. Pradeep et al [25] and chavan et al [26] reported birth asphyxia in $43 \%$ and $40.5 \%$ of neonatal deaths respectively. The incidence of prematurity was lower in our study i.e. $8.29 \%$.

Observation made above reveals that birth asphyxia is on the top of the list as a cause of neonatal mortality. This emphasizes the need for adoption of measures to prevent perinatal asphyxia and birth trauma. Similarly, infections need to be taken care by proper management of mothers having factors and babies born to such mothers.

\section{Conclusion}

The neonatal mortality in our study was $12.94 \%$. Since in our stud more than half of the neonatal deaths were associated with obstetric complications, birth asphyxia was on the top of the list followed by infection which was the major cause of neonatal mortality whereas meconium aspiration syndrome and septicemia were the main causes of neonatal morbidity. It is essential to augment our community health service for early detection and management of these problems. There is a need to establish level II neonatal care facility at district levels. We need to strengthen perinatal care, emergency services of obstetric services to enhance neonatal resuscitation.

Scope for further study: In order to find out the true neonatal morbidity and mortality, the study has to be planned in such a way that it conducts training programs for community health workers using simple and reliable methods, as we do not have adequate skilled staff to carry out such study in the community.

\section{Funding: Nil, Conflict of interest: Nil Permission from IRB: Yes}

\section{Reference}

1. Baba usmanahmadu ,Hammamisahbabba, jodaaisha Abdullah, Abraham musatizhe neonatal morbidity and mortality trenedina special care baby unit of a tertiary Hospital yola; the need to educate health workers and mothers on quality neonatal health practices [American journal of health research, Volume 1, issue 3 November 2013; 1 (3) : 99-103.

2. Kaushik S.L, Pamer V.R, Grover N and Kaushik R. neonatal mortality rate; relationship to birth weight and gestational age; Indian, j.pediatrics, 1998;65;429-433.

3. SinghM, Tripathy. K, Arya L. S; Birthweight gestational age correlates of neonatal mortality Indian j.pediatrics $1982 ; 49 ; 511$. 
4. Paul V. K, Singh M, Sundram K.R, Deorari A. KCorrelates of mortalityamong hospital born neonates with birth asphyxia. Natt Med j. Iandian1997;10 (54-57].

5. Klein J. perinatal morbidity and mortility associated with cesarean section 11 fetal salvage in the presence of stress factor obstet. Gynec,1959;20;1023.

6. Kwochi UE;Nduik. $\mathrm{N}$ wokoye IC..Amdi OF.O suorah DIC pattern of morbidity and mortality of newborn admitted into the sick and special care baby unit of Enugustate university teaching hospital.enugu state Nigerian journal of clinical practice May-June 2014-vol.7 issue 3 .

7. Hoang TTran, Lex Wdoyle ,KartherineJlee, Stephen M Graham. bA systemic review of the burden of neonatal mortality and morbidity in the Asian region WHOSouth - Eastjornal of public health 2012;1[3]; 239-248.

8. ClaciF. Weirich; Ana Lucla SS Andrade; Marilia Dalva Turchi Simonne A Silva, Otaliba L MoraisNeto; Ruth Ming misava; Solomar M Marques. neonatal mortality intensive care units of central Brazil. Rev. sauda public 39 2005; 39 [5]; 775-81.

9. Santosh K, Bhargav Umesh Bihari Lal, Arunkumar, Sudarshan Kumari \& Shanti ghosh, "morbidity patterns in newborns by gestationand intrauterine growth; Indian pediadrics, July 1974; vol .x1, no7.481-485.

10. Guptas; Chopra K Pramanick A; perinatal and neonatal morbidity in hospital born babies, Indian pediatric, 1972; 9; 586.

11. Shabbir Hussain; neonatal morbidity and mortality pattern in a tertiary care neonatal unit of a teaching hospital; Ann. pak. Inst Med. sci; .2014;10[1];7-11.

12. Singh M,Deorari, Khajuria R.C and Paul V.K A four years studyon neonatal morbidity in a new Delhihospital; Indian J. med. res [13] 94, June 1991; 186-192.

13. Bhakooo N, Narang A Kualkarni K. N Patils A. S; Banerjee C.K, WaliaBNS. Neonatal mortality and morbidity in hospital born babies. Indian paedidries, $1975 ; 12 ; 443-450$

14. Kapoor SK, Reddaiah VP,Lobo J,; Antenatal care and perinatal mortality' IndianJ. Pediatr, 1985; 52; 159162.

15. Ritu Rakholia,Vineet Rawat, Meharbanu Gurpreet singh. Neonatal morbidity and mortality of sick new born admitted in a teaching hospital of uttarakhand chrismed journal of health and research/vol 1/issue4/ oct-dec 2014.

16. Bhargava S.K.Lall. U.B. Kumar, H.K. Kumari S and Ghosh S. Morbidity pattern in new born by gestation and intrauterine growth. Indian pediatrics, $1974 ; 11 ; 481$.

17. Meherban singh, AK Deorari, RC khajuria and VK paul,'Perinatal \& Neonatal Mortality in an hospital", Indian J. Meds. Res (B) 94,feb.1991:1-5.

18. Abhay T. bang, Rani A. Bang, Sanjay Baitule, Mahesh Deshmukh and M. Hanimi Reddy. Burden of mortalities and Unmet need for health care in rural neonates. Indian Pediatrics, 2001;38;952-965.

19. Tallatseyal, FoziaHusnain, Asmaanwar Audit of neonatal morbidity and mortality at neonatal unit of Sir GangaramhospitalLahore annals vol 17, no. 1Jan-march 2011.

20. Gupta P. K., Gupta A.P. 1985, Perinatal mortality, Indian paediatrics 22;201-208.

21. Iman Seoud, Rasha M, Gamal El-din, Reem Nabilsaid; Heba Abou Hessin. predictors of neonatal mortality in intensive care unit in children hospital, Cairo university, Alexanderia Journal of pediatric volume 19, number 1, January 2005.

22. Choudhury panna. Thirupuram S. Gupta S. 'A study of maternal and neonatal factors in relation to perinatal mortality' Indian. pediatr,1978;15;313-318.

23. Mavalankar DV, Trivedi; C.R et al. levels and risk factors for perinatal mortality in Ahmedabad, Bull WHO, 1991; 69; 435-442 
24. KameswarmC, Bhatia BD, Bhat BV, et al. perinatal mortality. A hospital based study Indian pediatrics, 199, 30; 997-1001.

25. Pradeep M. Rajam L. Sudevan P. 'Perinatal mortality'. 'A hospital based study' Indian pediatr. 1995; 32’1091-1094
26. Chavan VS, DattalM.S, Khadilkar VV. Causes of early neonatalmortality.Indian pediatrics $992 ; 29$; 7817832 .

27. Misra PK. Bajpai PC. Tripathi T. Gupta k. and Katty D. Perinatal mortality. A hospital study. Indian pediatr.1973; 10;545.

\section{How to cite this article?}

Khuram SA, Prasad CN. Study of neonatal morbidity and mortality patterns in a referral tertiary care neonatal unit of a teaching hospital. Int J Pediatr Res.2016;3(8):589-596.doi:10.17511/ijpr.2016.i08.08 